
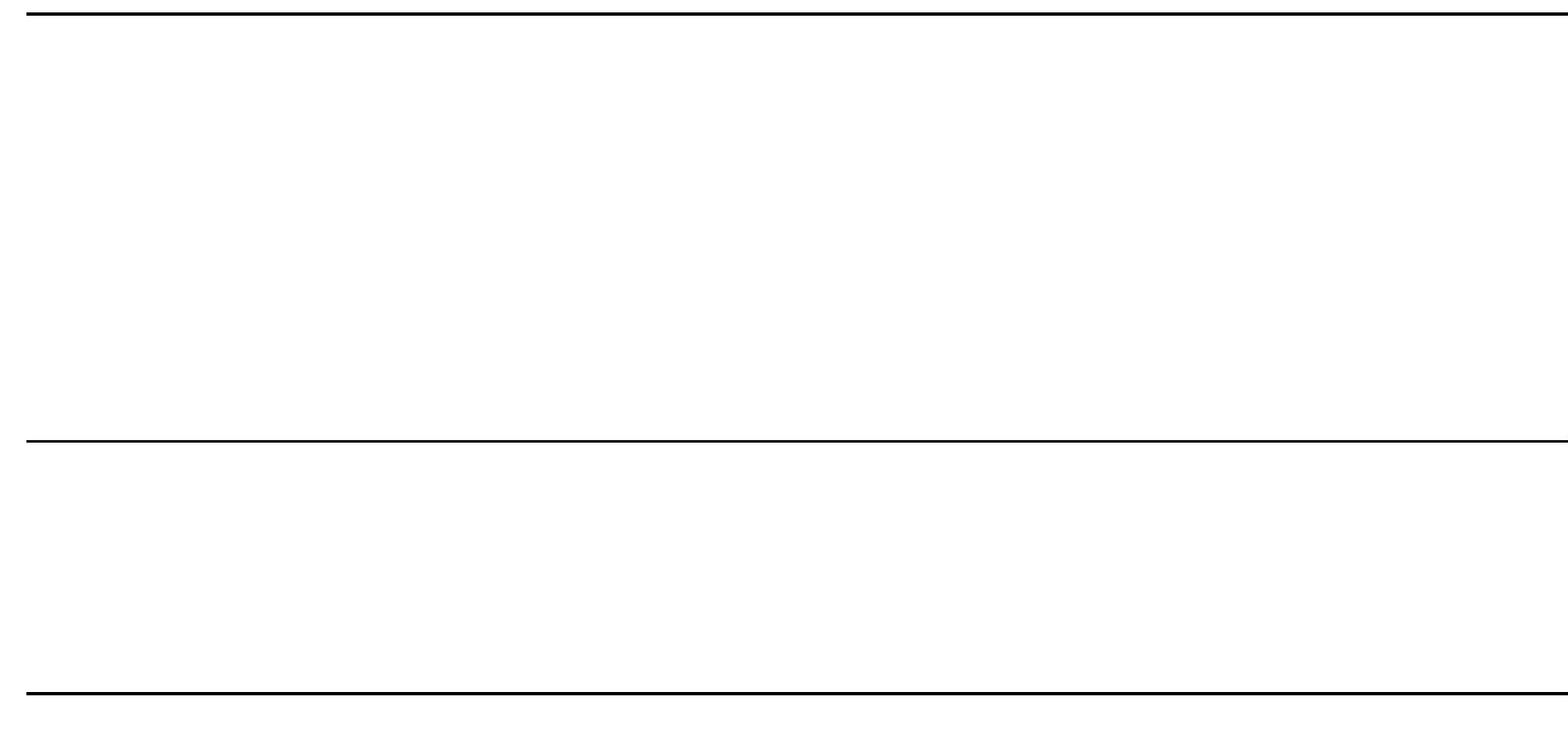


\title{
? nhanced serine production by bone metastatic breast cancer cells stimulates osteoclastogenesis
}

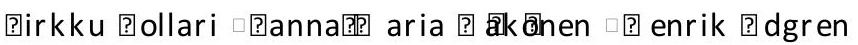 \\ ? ai?a ? olf $\square$ ? \\ Theresa ? uise $\square$ ? atthias ? ees $\square$ ? Ili ? allioniemi
}

Received: 20 October 2009/ Accepted: 13 March 2010

Springer Science+Business Media, LLC. 2010

\begin{abstract}
Since bone metastatic breast cancer is an incurable disease, causing signi $\sqsubset$ cant morbidity and mortality, an understanding of the underlying molecular mechanisms would be highly valuable. Here, we describe in vitro and in vivo evidences for the importance of serine biosynthesis in the metastasis of breast cancer to bone. We rst characterized the bone metastatic propensity of the MDA-MB-231(SA) cell line variant as compared to the parental MDA-MB-231 cells by radiographic and histological observations in the inoculated mice. Genome-wide gene expression pro $\square$ ling of this isogenic cell line pair revealed that all the three genes involved in the L-serine
\end{abstract}

[? lectronic supplementary material The online version of this article (doi:10.1007/s10549-010-0848-5) contains supplementary material, which is available to authorized users.

S. Pollari $(\&)$ P. Kohonen H. Sara M. Nees

Medical Biotechnology, VTT Technical Research

Center of Finland and Turku Center for Biotechnology,

Turku, Finland

e-mail: ext-sirkku.pollari $\square \mathrm{vtt}$. $\square$

\section{S.-M. Käkönen}

Institute of Biomedicine, Department of Anatomy,

University of Turku, Turku, Finland

H. Edgren M. Wolf O. Kallioniemi

Institute for Molecular Medicine Finland (FIMM),

University of Helsinki, Helsinki, Finland

T. Guise

Division of Endocrinology, Indiana University,

Indianapolis, IN, USA

Present Address:

S.-M. Käkönen

Amgen AB Finland, Espoo, Finland biosynthesis pathway, phosphoglycerate dehydrogenase (PHGDH), phosphoserine aminotransferase 1 (PSAT1), and phosphoserine phosphatase (PSPH) were upregulated in the highly metastatic variant. This pathway is the primary endogenous source for L-serine in mammalian tissues. Consistently, we observed that the proliferation of MDAMB-231(SA) cells in serine-free conditions was dependent on PSAT1 expression. In addition, we observed that L-serine is essential for the formation of bone resorbing human osteoclasts and may thus contribute to the vicious cycle of osteolytic bone metastasis. High expression of PHGDH and PSAT1 in primary breast cancer was signi $\sqsubset$ cantly associated with decreased relapse-free and overall survival of patients and malignant phenotypic features of breast cancer. In conclusion, high expression of serine biosynthesis genes in metastatic breast cancer cells and the stimulating effect of L-serine on osteoclastogenesis and cancer cell proliferation indicate a functionally critical role for serine biosynthesis in bone metastatic breast cancer and thereby an opportunity for targeted therapeutic interventions.

\section{? eywords Breast cancer Bone metastasis Osteoclast} L-serine

\section{?htr oduction}

Metastasis to bone is the most frequent cause of breast cancer morbidity and mortality. Currently available therapies are able to limit disease progression and alleviate painful symptoms but bone metastatic cancer remains incurable. In the metastatic process, tumor cells detach from the primary site, invade into blood vessels, and extravasate into the metastatic site. In order to form metastatic lesions, tumor cells need to adapt to the bone 
microenvironment and interact with the host cells. The majority of the skeletal lesions in breast cancer are osteolytic, characterized by enhanced osteoclast activity and bone destruction. Cancer cells adapt to the bone microenvironment and start a vicious cycle causing bone destruction. Several cancer cell-derived factors, such as parathyroid hormone-related peptide (PTHrP), have been shown to contribute to this process $\square, 2 \square$ Bone is a rich source of growth factors, including transforming growth factor (TGF) and, insulin-like growth factors (IGFs), and $\square$ broblast growth factors (FGFs), which are released as a consequence of osteoclastic bone resorption and can stimulate cancer cell survival and production of more osteolytic factors $3 \square$ However, in order to develop more effective treatment strategies, a comprehensive understanding of the critical molecular mechanisms involved in the bone metastatic process is needed.

In vivo modeling of bone metastatic breast cancer is limited by the fact that spontaneous bone metastases are rare in animals. The MDA-MB-231 breast cancer cell line is commonly used to model breast cancer bone metastasis in vivo. This cell line was derived from the pleural effusion of an estrogen receptor negative, late-stage breast cancer patient. When MDA-MB-231 cells were inoculated into the blood circulation of immunode cient mice by intracardiac inoculation, osteolytic lesions were formed $4 \square$ MDA-MB231 variants with enhanced bone metastatic abilities have been created, compared, and used for studying molecular mechanisms of bone metastasis 5, 6ロ The MDA-MB231(SA) variant has been used in several studies $\square, 8 \square$ and is known to be highly bone metastatic. However, comparative molecular studies on this variant and the parental MDA-MB-231 cell line have not been published. Here, we compared the in vivo bone metastatic properties between the MDA-MB-231(SA) variant and parental MDA-MB231 cells and then used genome-wide gene expression pro $\square$ ing to identify molecular mechanisms that could explain the different metastatic properties of the two cell types.

\section{? aterials and methods}

Breast cancer cell lines and culture conditions

The parental MDA-MB-231 cancer cell line was obtained from the American Type Culture Collection (ATCC). MDA-MB-231(SA) cells were spontaneously derived from these cells during a long in vitro culture. Both cell types were maintained in low-glucose DMEM with $10 \%$ inactivated fetal bovine serum (FBS), $1 \%$ nonessential amino acids, $100 \mathrm{U} / \mathrm{ml}$ penicillin, and $100 \mathrm{~g} / \mathrm{ml}$ streptomycin in an incubator with a humidi $\sqsubset$ ed atmosphere of $95 \%$ air and $5 \% \mathrm{CO}_{2}$ at $37 \mathrm{C}$.

\section{Mouse experiments}

The animal protocols were approved by the Institutional Animal Care and Use Committee at the University of Texas Health Science Center in San Antonio and were in accordance with the National Institutes of Health Guide for the Care and Use of Laboratory Animals. 100,000 MDAMB-231(SA) or parental MDA-MB-231 cells were inoculated into the left cardiac ventricle of anesthetized $(30 \%$ ketamine and $20 \%$ xylazine in $0.9 \% \mathrm{NaCl}$ ) 5 -week-old female athymic nude mice (Balb/c nu/nu, Harlan, Indianapolis, IN) (n $\square 7$ mice/cell line) as described previously 2, 4卬 Body weight of the mice was monitored during the experiment, and the mice were killed when they became cachectic, or paraplegia was observed. The development of bone metastases was monitored weekly or bi-weekly by $\mathrm{X}$-ray radiography. Animals were anaesthetized with Ketamine-Xylazine cocktail and X-rayed in a prone position using a 43855A X-ray system (Faxitron, Lincolnshire, IL, USA), as described previously $2 \square$ The total area and number of lesions in hind (left and right tibia and femur) and fore (left and right humerus) limbs per mouse were quanti $\sqsubset$ ed from the images using image analysis software (Java, Jandel Video Analysis; Jandel Scienti $\sqsubset$ c, Corte Madera, CA, USA). All of the radiographs were evaluated without knowledge of the treatment groups. Heart, lungs, spleen, kidneys, liver, and adrenal glands were collected when killed for histology to determine possible soft tissue metastasis in addition to the bone metastasis. Tissue sections $(4 \mathrm{~m})$ were cut and stained with hematoxylin and eosin ( $\mathrm{H} \square \mathrm{E})$, orange $\mathrm{G}$ and phloxine $\mathrm{B}$ using standard protocols.

Genome-wide comparative genomic hybridization

Array-based CGH was performed using Agilent 244K oligonucleotide microarrays according to the direct method of the June 2006, version 4 protocol (Agilent Technologies). Female genomic DNA (Promega) was used as reference in both hybridizations. Labeling of $3 \mathrm{~g}$ of digested and puri $\llbracket$ ed tumor and reference DNA was performed with Cy5-dUTP and Cy3-dUTP (Perkin-Elmer), respectively, according to the protocol. Labeled tumor and reference samples were pooled and hybridized onto an array. The arrays were washed and scanned with a laser confocal scanner (Agilent Technologies). Signal intensities were extracted using the Feature Extraction software (Agilent Technologies). CGH Analytics (Agilent Technologies) was used for initial data analysis and visualization. 
Gene expression pro $\square$ ing

Gene expression levels were measured using the Affymetrix GeneChip U133 Plus 2 system (Affymetrix). RNA was extracted from the parental MDA-MB-231 and MDA-MB231(SA) cells using the RNeasy kit (Qiagen) according to the manufacturer's instructions. Sample processing and labeling were performed according to the protocol provided by Affymetrix. We used $3 \mathrm{~g}$ of total RNA for the initial cDNA synthesis. Chips were scanned using the GeneChip Scanner 3000 (Affymetrix). The raw cel $\square$ les were processed separately using the $\mathrm{R}$ language $9 \square$ and the RMA method implemented in the Bioconductor $\square 0$ package affy $11 \square$ Alternative CDF $\square$ les mapping Affymetrix probes directly to Ensembl gene IDs were used in the preprocessing $12 \square$ The microarray data were deposited in the Gene Expression Omnibus (GEO, http://www.ncbi. nlm.nih.gov/geo, accession number GSE20085).

\section{Western blot analyses}

Cells were lysed in SDS buffer (containing 30\% glycerol, $3 \%$ SDS, 3\% -mercaptoethanol, $0.015 \%$ bromophenol blue, and $0.19 \mathrm{M}$ Tris- $\mathrm{HCl}(\mathrm{pH} 6.7)$ ). Lysates were incubated at $95 \mathrm{C}$ for $5 \mathrm{~min}$ and then loaded into an SDSPAGE gel. Separated proteins were transferred to a nitrocellulose membrane and immunoblotted using chicken anti-human PSAT1 antibody (US Biological, Swampscott, MA, USA), rabbit anti-human PSAT1 antibody (Strategic Diagnostics Inc., Newark, DE, USA), mouse anti-human PHGDH antibody (Abnova, Taipei, Taiwan), mouse anti-actin (Sigma) or rat anti-Hsc70 antibody (Stressgen, Canada). Secondary antibodies: HRP-conjugated donkey anti-chicken IgY (Jackson ImmunoResearch, West Grove, PA, USA), IRDye 800-conjugated anti-rabbit IgG (Rockland Immunochemicals, Gilbertsville, PA), Alexa Fluor 680 goat anti-mouse IgG (Invitrogen), HRP-conjugated goat anti-rat IgG (GE Healthcare). The Amersham enhanced chemiluminescence system (GE Healthcare) was used for chemiluminescent imaging, and Odyssey Licor device for uorescence imaging of proteins.

\section{Quantitative real-time RT-PCR}

Total RNA was isolated from cells using RNeasy Mini kit (Qiagen). Total RNA weighing 300 ng was reverse transcribed using the High Capacity cDNA Reverse Transcription kit (Applied Biosystems, Foster City, CA, USA). The primers and probes speci $\sqsubset \mathrm{c}$ for each target gene or the endogenous control ACTB ( -actin) were designed by the the ProbeFinder software (Roche Applied Biosciences, Basel, Switzerland) and are listed in the Online Resource 1 in Electronic supplementary material. The cDNAs were diluted 1/10, and TaqMan quantitative real-time reverse transcription PCR (RT-PCR) was performed using the Applied Biosystems 7900HT instrument. The results were analyzed using the SDS 2.3 and RQ manager softwares (Applied Biosystems, Foster City, CA, USA), and the expression of target mRNA was determined by relative quantitation using -actin as an endogenous control.

\section{Generation of shRNA expressing cells}

MDA-MB-231(SA) cells were transduced with lentiviral particles containing an shRNA sequence targeting PSAT1 (Sigma) or a scrambled shRNA sequence not targeting any human gene (Sigma). Transduction was performed in the presence of $8 \mathrm{~g} / \mathrm{ml}$ hexadimethrine bromide. Transduced cells were selected with $4 \mathrm{~g} / \mathrm{ml}$ puromycin. PSAT1 knockdown was con $\sqsubset$ rmed using quantitative RT-PCR and Western blotting.

\section{Cell proliferation assay}

In order to remove the amino acids, inactivated FBS was dialyzed against PBS using a dialysis cassette with a molecular weight cut-off of 2,000 at $4 \mathrm{C}$ for $29 \mathrm{~h}$. Cells were plated in 96-well plates in Minimum Essential Medium (MEM) (Invitrogen) with 10\% dialyzed FBS, $100 \mathrm{U} / \mathrm{ml}$ penicillin, and $100 \mathrm{~g} / \mathrm{ml}$ streptomycin. L-serine (Sigma) was added to the wells at speci $\square$ c concentrations indicated in the gure legends. Each condition was tested in three replicates. The cells were incubated in a humidi $\sqsubset$ ed atmosphere of $95 \%$ air and $5 \% \mathrm{CO}_{2}$ at $37 \mathrm{C}$. Cell viability was measured using the CellTiter-Blue Reagent (Promega) according to the manufacturer's instructions.

\section{Osteoclast culture}

In order to remove the amino acids, FBS (Osteoclast Medium BulletKit, Lonza) was dialyzed against PBS using a dialysis cassette with a molecular weight cut-off of 2,000 at $4 \mathrm{C}$ for $27 \mathrm{~h}$. A total of 10,000 human osteoclast precursor cells (Lonza) were seeded onto bovine bone slices (Nordic Bioscience Diagnostics, Herlev, Denmark) in 96well plates and cultured in an incubator with a humidi $\llbracket$ ed atmosphere of $95 \%$ air and $5 \% \mathrm{CO}_{2}$ at $37 \mathrm{C}$. The culture conditions for the $\square$ rst 7 days of culture were as follows: MEM (Invitrogen) with 9\% dialyzed FBS and following supplements from the Osteoclast Medium BulletKit (Lon$\mathrm{za}): 2 \mathrm{mM}$ L-glutamine, $100 \mathrm{U} / \mathrm{ml}$ penicillin, $100 \mathrm{~g} / \mathrm{ml}$ streptomycin, $26.6 \mathrm{ng} / \mathrm{ml} \mathrm{M-CSF}$, and $53.2 \mathrm{ng} / \mathrm{ml}$ soluble RANKL. The $\mathrm{pH}$ of the medium was adjusted to 7.4. L-serine (Sigma) or O-phospho-DL-serine (Sigma) was added to the culture wells at speci $\sqsubset \mathrm{c}$ concentrations indicated in the gure legends for each experiment. After 7 
days of culture, the medium was collected and replaced with MEM with $9 \%$ dialyzed FBS, 2 mM L-glutamine, $100 \mathrm{U} / \mathrm{ml}$ penicillin, $100 \mathrm{~g} / \mathrm{ml}$ streptomycin, $33 \mathrm{ng} / \mathrm{ml}$ recombinant human M-CSF (R $\square \mathrm{D}$ Systems), and $66 \mathrm{ng} / \mathrm{ml}$ recombinant human soluble RANKL (PeproTech EC, London, UK). The $\mathrm{pH}$ of the medium was adjusted to 6.9. L-serine or O-phospho-DL-serine was added to the culture wells at speci $\square$ c concentrations indicated in the gure legends for each experiment. Each condition was tested in at least four replicates. After 11 days of culture, medium samples were collected. The cells were $\square$ xed with 3\% PFA and stained for tartrate-resistant acid phosphatase (TRACP) using the Acid Phosphatase Leukocyte Kit (Sigma) according to the manufacturer's instructions.

\section{Immunoassays}

Tartrate-resistant acid phosphatase (TRACP) 5b activity was measured from the medium samples using a speci $\square \mathrm{c}$ immunoassay (BoneTRAP Assay, Immunodiagnostic Systems Ltd, Boldon, UK) after 7 days of culture. Bone resorption was quanti ed by measuring the concentration of the degradation products of C-terminal telopeptides of type I collagen (CTX) in the conditioned medium using the CrossLaps for Culture ELISA kit (Nordic Bioscience Diagnostics) after 11 days of culture.

Analysis of gene expression in clinical breast cancer samples

Association of PHGDH, PSAT1, PSPH, and SLC1A4 expression with time to relapse in the Affymetrix gene expression studies GSE6532 and GSE12276 (n $\mathrm{n}$ 368) and with overall survival in the Affymetrix gene expression studies GSE3494 and GSE1456 (n 0 393) was assessed by Kaplan-Meier analysis. Correlation of differential expression of PHGDH, PSAT1, PSPH, and SLC1A4 with clinical features was analyzed using a data set of 251 breast tumor samples hybridized to Affymetrix U133A and U133B human GeneChips (GSE3494) $13 \square$ U133A and U133B chips were preprocessed separately using the $\mathrm{R}$ language $9 \square$ and the RMA method implemented in the Bioconductor $\square 0 \square$ package affy $\square 1 \square$ Alternative CDF $\square$ les mapping Affymetrix probes directly to Ensembl gene ids were used in preprocessing $12 \square$. Data from U133A and U133B were combined by calculating the medians of genes appearing on both array types. TP53 status is based on the classi $\llbracket$ er developed by Miller et al. $13 \square$ Breast cancer subtypes were assigned to samples based on expression data $\square 4 \square$. Ki67, PCNA, and ERBB2 status was likewise estimated from the expression data itself.
Statistical analysis

The results are reported as mean $\square$ SD. Comparison between the groups in the in vivo experiment was done using the one way repeated measures ANOVA (body weight, lesion area, lesion number) or by a log-rank test (survival). Statistical signi $\sqsubset$ cance of the immunoassay and cell proliferation data were analyzed using an unpaired Student's t test. Statistical signi $\sqsubset$ cance of survival differences for breast cancer patients was determined by a logrank test between the highest quartile and the remaining three quartiles with respect to the gene expression values.

? esults

Bone metastatic activity of MDA-MB-231(SA) and parental MDA-MB-231 cells in vivo

Owing to the tumor-induced cachexia and paraplegia, all mice inoculated with MDA-MB-231(SA) cells were killed on or before day 26 after inoculation (survival $23 \square$ 4.2 days, mean $\square$ SD). The mean body weight of the MDA-MB-231(SA) group was signi $\square$ cantly smaller already on day 21 when compared to the parental MDA-MB-231 group (Fig. 1a). The $\square$ rst mouse in the parental MDA-MB231 group was killed on day 58, and the mean survival time in this group was 76.5 19.1 days (mean $\square$ SD) (Fig. 1b). None of the mice in this group was paraplegic when killed. Clear osteolytic lesions were observed in six out of seven mice in the MDA-MB-231(SA) 2 weeks after inoculation and the lesions were severe when killed (Fig. 1c, Online Resource 2 in Electronic supplementary material). Histology revealed no soft tissue metastasis. Only one mouse in the parental MDA-MB-231 group had a clear osteolytic lesion, which was visible in X-ray on day 56. However, $\square$ ve mice in this group had soft tissue tumors according to autopsy and histology.

Genes involved in L-serine biosynthesis or transport are overexpressed in the highly bone metastatic MDA-MB-231(SA) cells

We used array-based comparative genomic hybridization (CGH) to compare the highly metastatic MDA-MB231(SA) variant and the parental cell line. This analysis showed that the genetic aberrations were highly similar in the two cell lines (Online Resource 3 in Electronic supplementary material). We then compared the genome-wide gene expression pro $\square$ les of the highly metastatic and parental MDA-MB-231 cells. Only $1.7 \%$ (315 genes) of all genes were over 2.5-fold upregulated and 1.1\% (198 genes) over 2.5-fold downregulated in the highly metastatic 
?]ig. 1 Bone metastatic activity of MDA-MB-231(SA) and parental MDA-MB-231 cells in mice. a Change in body weight of the mice as a function of time after inoculation. b Survival of the mice inoculated with MDAMB-231(SA) or parental MDAMB-231 cells. c Radiographic analysis of the osteolytic lesions. N.D. not determined because the mice inoculated with MDA-MB-231(SA) cells had already been killed before this time point. $\square \mathrm{P} \quad 0.05$, $\square \mathrm{P} \quad 0.01, \square \square \mathrm{P} \quad 0.001$, signi $\sqsubset$ cantly different from the mice inoculated with parental MDA-MB-231 cells variant. We used the DAVID functional annotation tool $\square 5 \square$ and WebGestalt gene set analysis toolkit $\square 6 \square$ to assess enrichment of gene ontology (GO) and pathway associations for the upregulated genes. These analyses indicated highest enrichment for organic acid metabolic process ( $P \square 1.3 \square 10^{\square 6}$ ), amino acid and derivative metabolic process $\left(P \square 1.7 \square 10^{\square 6}\right.$ ), amine metabolic process ( $P \square 2.6 \square 10^{\square 5}$ ), and nitrogen compound biosynthetic process ( $P \square 5.1 \square 10^{\square 4}$ ) (Online Resource 4 in Electronic supplementary material). The most signi $\square$ cantly enriched KEGG pathway was Glycine, serine, and threonine metabolism ( $P \square 0.0079$ ) (Online Resource 5 in Electronic supplementary material). Three genes encoding the enzymes that catalyze the steps of the phosphorylated pathway of L-serine biosynthesis (Fig. 2), phosphoglycerate dehydrogenase (PHGDH), phosphoserine aminotransferase 1 (PSAT1), and phosphoserine phosphatase (PSPH) are involved in all of the above mentioned enriched GO categories and in the most signi $\square$ cantly enriched pathway, and upregulated (5.1-, 5.8-, and 2.6-fold, respectively) in the highly metastatic MDA-MB-231(SA) cells. Furthermore, a transporter for serine, alanine, cysteine, and threonine, SLC1A4 (solute carrier family 1 (glutamate/neutral amino acid transporter), member 4) was 3.4-fold upregulated in the MDA-MB-231(SA) cells. Overexpression of PHGDH, PSAT1, PSPH, and SLC1A4 mRNA was conrmed by quantitative RT-PCR and overexpression of PHGDH and PSAT1 at protein level by Western blotting (Fig. 3). ?ig. 2 Phosphorylated pathway of L-serine biosynthesis

Expression of genes involved in L-serine biosynthesis or transport in in vivo selected bone metastatic MDA-MB-231 variants

Kang et al. $5 \square$ pro $\square$ led gene expression (Affymetrix U133A) in 12 in vivo selected MDA-MB-231 subpopulations with different bone metastatic abilities and in the parental MDA-MB-231 cells. We used the data provided by Kang et al. and compared the expression of $\mathrm{PHGDH}$, PSAT1, PSPH, and SLC1A4 between the strongly ( $\mathrm{n} \square$ 6) and weakly ( $\mathrm{n} \square$ ) metastatic cells. Because the gene expression data were not publicly available in raw cel format and therefore not applicable to the normalization method described above, we used the data at probe set level 
- 
